\title{
The impact of probiotics in obese pregnancy to reduce maternal fasting glucose: A randomized controlled trial
}

\author{
K. L. Lindsay ${ }^{1}$, M. Kennelly ${ }^{1}$, M. Culliton ${ }^{2}$, O. C. Maguire $^{3}$, T. Smith ${ }^{3}$, F. Shanahan ${ }^{4}$, \\ L. Brennan ${ }^{5}$ and F. M. McAuliffe ${ }^{1}$ \\ ${ }^{1}$ UCD Obstetrics and Gynaecology, School of Medicine and Medical Science, University College Dublin, National \\ Maternity Hospital, Dublin 2, Ireland, ${ }^{2}$ Laboratory Medicine, National Maternity Hospital, Dublin 2, Ireland, \\ ${ }^{3}$ Department of Clinical Chemistry, St. Vincent's University Hospital, Elm Park, Dublin 4, Ireland, ${ }^{4}$ Department of \\ Medicine, Alimentary and Pharmabiotic Centre, University College Cork, Cork, Ireland and ${ }^{5}$ Institute of Food and \\ Health, UCD School of Agriculture and Food Science, University College Dublin, Belfield, Dublin 4, Ireland
}

\begin{abstract}
Probiotics are live microorganisms which, when consumed in adequate amounts, may confer a health benefit on the host ${ }^{(1)}$. Recent studies have reported beneficial effects of probiotics on maternal glycaemia amongst healthy pregnant women ${ }^{(2,3)}$. Obesity significantly increases the risk of impaired glucose tolerance in pregnancy but the glycaemic effects of probiotics in this specific obstetric group requires further investigation. The aim of this study was to investigate the effects of a probiotic capsule on maternal fasting glucose, other metabolic indices and obstetric outcomes in obese pregnant women.

This double-blind placebo-controlled randomized trial recruited 175 non-diabetic pregnant women with a body mass index of $30.0-39 \cdot 9 \mathrm{~kg} / \mathrm{m}^{2}$ from antenatal clinics in the National Maternity Hospital, Dublin. Women were randomized to either a daily probiotic (Lactobacillussalivarius UCC118) or placebo capsule from 24 to 28 week's gestation. The primary outcome was change in fasting glucose between the groups from pre- to post-intervention. Secondary outcomes were incidence of gestational diabetes and neonatal anthropometry. A sample size of 100 was required to detect a difference in fasting glucose of $0.4 \mathrm{mmol} / \mathrm{l}$. Results were analysed on an intention-to-treat basis using General Linear Model, adjusting for BMI.

Among 138 women who completed the study (63 probiotic, 75 placebo), mean early-pregnancy BMI was $33 \cdot 6 \mathrm{~kg} / \mathrm{m}^{2}$ (SD 2.6), which differed significantly between probiotic $\left(32.9 \mathrm{~kg} / \mathrm{m}^{2}\right)$ and placebo $\left(34 \cdot 1 \mathrm{~kg} / \mathrm{m}^{2}\right)$ groups. Adjusting for BMI, the change in maternal fasting glucose did not differ significantly between the treated and control groups $(-0.09$ [SD 0.27] vs. $-0 \cdot 07$ [SD 0.39] $\mathrm{mmol} / 1 ; p=0 \cdot 391 ; \mathrm{B}=-0 \cdot 05 ; 95 \% \mathrm{CI}-0 \cdot 17$ to $0 \cdot 07)$. There were also no differences in incidence of impaired glycaemia ( $16 \%$ probiotic group vs. $15 \%$ placebo group, $p=0.561)$, mean birth weight (3.70 $\mathrm{kg}$ [SD 0.52] probiotic group vs. $3.68 \mathrm{~kg}$ [SD 0.51] placebo group, $p=0.723$ ), other metabolic parameters (maternal insulin, c-peptide, HOMA, lipids) or pregnancy outcomes (preeclampsia, caesarean section, large for gestational age, admission to neonatal intensive care unit).

While previous studies of probiotics in healthy pregnant women showed some beneficial glycaemic effect, this randomized trial demonstrated no impact on fasting glucose, obstetric or neonatal outcomes in obese pregnancy.
\end{abstract}

This work was supported by the National Maternity Hospital Medical Fund and Ivo Drury Award.

1. Report of a joint FAO/WHO expert consultation on evaluation of health and nutritional properties of probiotics in food including powder milk and live lactic acid bacteria. Cordoba, Argentina: FAO/WHO, 2001.

2. Lindsay KL, Walsh CA, Brennan L, et al. (2013) J Mat FetalNeonat Med 26, 772-778.

3. Laitnen K, Poussa T, Isolauri E, et al. (2009) Br J Nutr 101, 1679-1687. 